\title{
Information Systems Planning - How to enhance creativity?
}

\author{
Vitor Santos ${ }^{1}$, Luís Amaral ${ }^{1}$, Henrique Mamede ${ }^{2}$ \\ ${ }^{1}$ Universidade do Minho, Guimarães, Portugal \\ ${ }^{2}$ Universidade Aberta, Lisboa, Portugal \\ vsantos@dsi.uminho.pt, amaral@dsi.uminho.pt,hsmamede@univ-ab.pt
}

\begin{abstract}
The global competitiveness and the organizations ability to make effective use of information technology and to focus on innovation and creativity are recognized as being important. The perspective of using creativity techniques or some adaptations, to help innovation in the area of information systems seems to be promising.

In this article we propose a strategy for the introduction of creativity in the information systems planning in order to build more agile and efficient information systems, allowing therefore more competitive business.
\end{abstract}

Keywords: Information Systems Planning, Creative Thinking, Innovation

\section{Introduction}

The ability of companies effectively using information technologies and betting on innovation and creativity is recognized today as one important factor on the competiveness and agility of the companies. These take natural benefits through creativity and innovation by restructuring their processes, projects and products [5]. The Information Systems Planning (ISP) is a vital activity for the success and competitiveness of companies [3].

The diversity of the sectors in corporate activity, the different contexts and organizational structures are, along with the growing complexity of the globalized world of business, a huge challenge for the effectiveness of this project.

In this context, the chances of resourcing to known creativity techniques or their adaptations, in order to mediate the spawning of ideas, help produce new combinations, supply unexpected answers, as well as original, useful and satisfactory, in the area of Information Systems is challenging.

In this article, in a more limited aspect, we propose a method for the introduction of creativity and innovation techniques in the Information Systems Planning, regarding 
the construction of more agile and efficient Information Systems, which consequently allow an enhanced corporate competitiveness.

\section{Creativity in the Information Systems Planning}

The role of Information Systems Planning (ISP) has become crucial in the development and implementation of effective strategic plans in the organizations [9]. The growing uncertainty in the markets has led the companies to be more pro-active and to develop strategies that might allow enhancing their competitiveness. The strategy formula is oriented through company's mission and objectives based on a careful analysis of the involving means and the company itself, as shown in Figure 1.

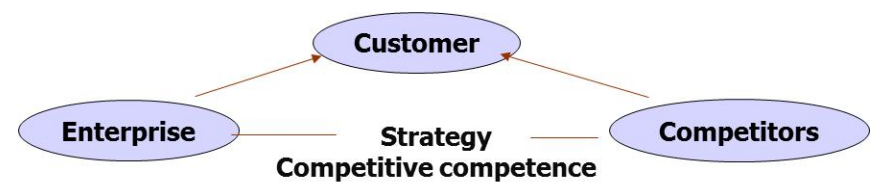

Figure 1 - Strategy and competitiveness

Information technology allows a set of opportunities to reach a competitive edge and to adjust the Information Systems to the company's benefit. On the other hand, organizations verify that the capacity do provide a quick answer to unforeseeable events is fundamental to its survival [1].

In spite of the importance of the creativity in the Information Systems Planning being acknowledged, and a part of the main approaches to ISP as for instance the threestage model by Bowman [4] and the multidimensional approach of Earl [7], developed investigation in this area has been scarce. Ruohonen and Higgins analyze the potential of ISP's activity theory application [12]. The analysis is divided into three separate time periods according to an ISP evolutionary perspective, and in each of the time periods the relation of creativity with Information Systems Planning is discussed.

Horton and Dewar propose the use of alexandrine patterns formalism to encapsulate the creative aspects of strategic Information Systems formation [8]. They apply a case-study on a Great Britain police force that allows them to derivate two patterns which demonstrate the use of a creative practice on a micro political level.

In the theoretical review on Information Systems Creativity, in particular in this article's context it stands out, on one hand, the importance of ISP activity in the definition of systems to develop, in order that these be aligned with the organization's strategy, and on the other hand, the need that this activity be creative and innovating thus providing the development of more competitive and adaptable solutions to environmental conditions. 
Regarding the revision made on creativity and its techniques, it was possible to come to the conclusion it is a solid area, with about two hundred different creative techniques that can be grouped and used in different situations. Of these, that may support, stimulate, accelerate the creative production, we select for the use in different stages of the method, those that prove more adequate in which case.

Crossing these two areas of study, Information Systems and creative thought, there are various investigation themes, primary ones being: generating computer mediated ideas, creativity in the development of Information Systems, support tools to creativity and creative Information Systems, as well as Information Systems Planning creativity. It is on this last theme of crossing between Information Systems and creative thought that the present proposal fits in. More specifically in the convergence between ISP that, on its own should be creative, with an investigation on creativity and creative thought.

\section{A strategy to introduce creativity in ISP}

As there are several approaches to ISP, where Alignment and Impact stand out, it is important to understand which the introduction and creative mechanisms are in these. Since Bowman's three stage model and Earl multidimensional are the more representative on Alignment and Impact approaches, and the PRAXIS/a by Amaral [2] an important conjugation of the three stage model and multidimensional approach, we focus our investigation on these three approaches.

The «Three Stage Model» [4] is one of the most relevant ISP approaches. It is based on the alignment of IS with the organization, caring for the analysis and needed information as well as resources rationalization. It follows a top-down strategy and it points out a range of ordained and well defined activities and tasks.

In this approach ISP activities are done in three separate stages. Figure 2 describe these stages according to their main activities, linkage and main results. 


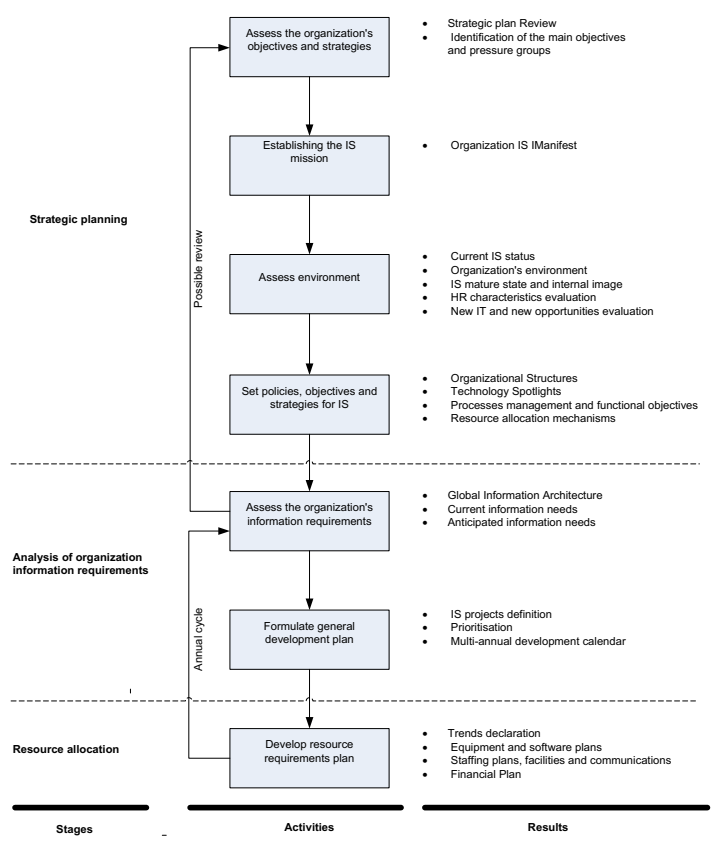

Figure 2 - Three Stage Model

Earl, on his «Multidimensional Approach» [7], defends that ISP must separately seek these three goals: to clarify the needs and strategy of the organization relating to its IS, assess the support to the organization and current IS usage, and Innovate by taking advantage of given strategic opportunities by IT/IS. This demand must be done in separate processes, but they must reflect on each other. The reason the search must be done separately is due to the fact that each goal is totally separate and has unique characteristics. Earl called «legs» to each of these distinct research processes. In Figure 3, the characteristics and main focuses of each «legs» are described.

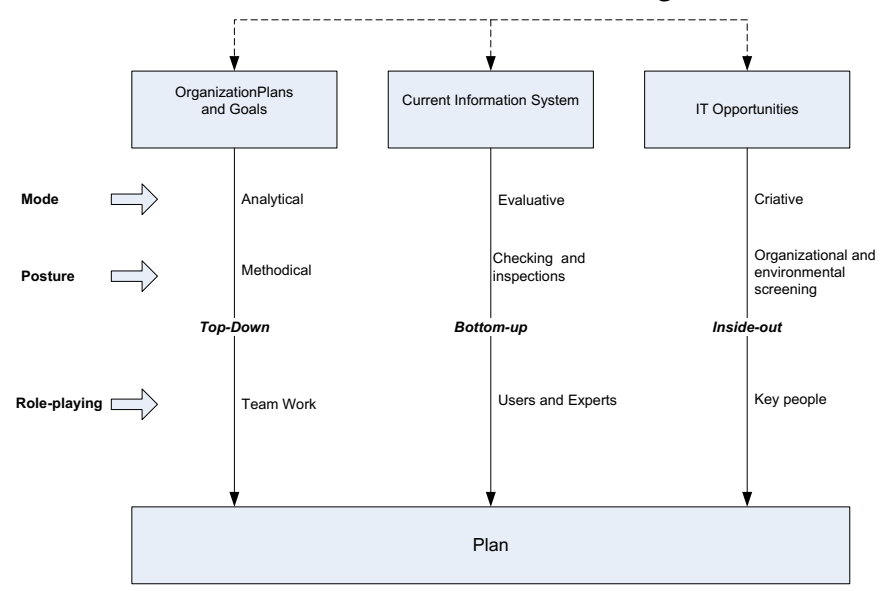

Figure 3 - Multidimensional Approach - adapted from Amaral [2] 
The PRAXIS/a approach, as visualized on Figure 4, incorporates simultaneously the «Multidimensional Approach» and «Three Stage Model», and complements them with intention or focus: while the first seeks the alignment and impact of IT/IS in the organization, the second seeks the IT/IS alignment with the organization and linkage of the ISP with the Information Systems Development (ISD).

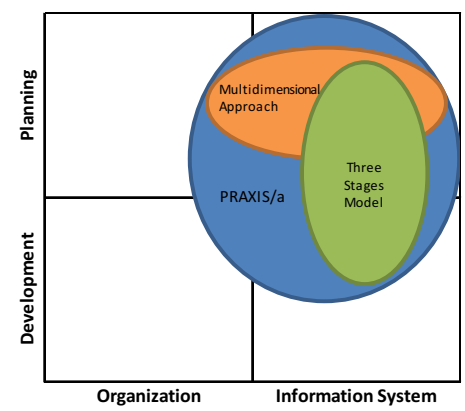

Figure 4 -PRAXIS/a approach - Three Stages Model and Multidimensional Approach relative positioning - adapted from Amaral [2]

The strategy we propose aims at incorporating creative processes and creativity techniques in different moments of main ISP approaches. For that we recognize the differences between approaches and phases, where to each one will make sense to introduce creative processes, as illustrated in the zones identified with $« \mathrm{C} »$ on Figure 5 .

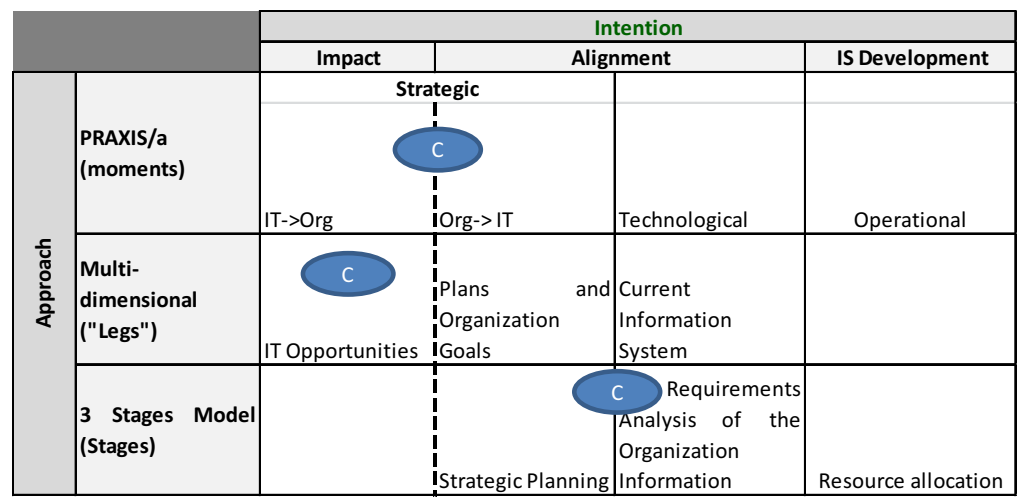

Figure 5 - Creativity in ISP approaches

To turn the creative processes viable, we propose a generic introduction and creativity method, to adapt according to the specifics of each of the three approaches.

This method resorts to several creativity techniques that we think are adequate to the different stages of the method, and in its whole allow it creative power. The method is 
based on six stages. Figure seven presents a global view on the method, in which stages to execute in sequence are now described:

Stage 1- Building a Team- In this stage we proceed to build a team that will apply the method. It is important to define a team leader and identify the competences and personal experience of each member. Preferably team members should have different personal and professional profiles.

Stage 2- Clarifying the Goal- In this stage the team should, starting with a generic business need (typically new challenge, opportunity, lacking or improvement) clearly identify the objective to be reached by the Information System to develop. Out of the need of the initial business necessity, the goal must be defined in an effective, clear, precise and measurable way.

Stage 3 - Understanding the needs of the Organization- After a clear definition of the goal, we must examine in depth the actual organization situation facing the objective to attain. This analysis means to comprehend which points are condition to success and precise what the ideal solution will be, under the point of view of achieving the goal. On this last subject, using creative techniques, as brainstorming, may be very useful, for instance to «define the ideal goal to reach» in unexpected and innovative ways.

Stage 4 - Focus on primary causes- This stage has for goal identifying the primary causes in the origin of the business. Root cause analysis (RCA) is a class of problem solving methods aimed at identifying the root causes of problems or events. The objective in root cause problem solving is to discover the points of leverage where patterns of behavior originate and can be changed.

This is a fundamental issue, for it will only be possible to think of new approaches and solutions having established the true causes in the origin of business necessities. Here too, resorting to creativity techniques, like brainstorming can prove very useful to identify deeper causes.

Stage 5- Finding Opportunities for Information Systems (OIS) - In this stage, considering the needs of the organization, and the primary causes, one or more creativity techniques are applied, to be selected according to typology in cause (as explained in Figure 6), in the attempt to obtain innovative solutions to address these causes.

\begin{tabular}{|c|c|c|c|c|c|}
\hline & \multicolumn{4}{|c|}{ OIS source } \\
\hline & & No Coverage & Improvement & New business & Integration \\
\hline \multirow{6}{*}{ 产 } & Idea Box & & $x$ & & $X$ \\
\hline & Brainstorming & $X$ & & $x$ & $x$ \\
\hline & Brute Thinking & & & $X$ & \\
\hline & Reversal & & & $\mathrm{X}$ & \\
\hline & SCAMPER & & $X$ & & $X$ \\
\hline & White Board & & & $\mathrm{X}$ & \\
\hline
\end{tabular}

Figure 6 - Application of creativity techniques to identify innovative Opportunities for Information Systems (OIS) 
In this stage it is important to think creatively and keep an open mind so it is possible to get the maximum of alternate solutions. Given the foreseeable elevated number of alternate solutions, one should use a formal method to choose the better solution.

Stage 6-Incorporating OIS in the Plan- Finally in the last stage, one will to proceed to incorporate the Opportunities for Information Systems found in Stage 5 in the plan. The plan must be built according to the structure adopted by the organization.

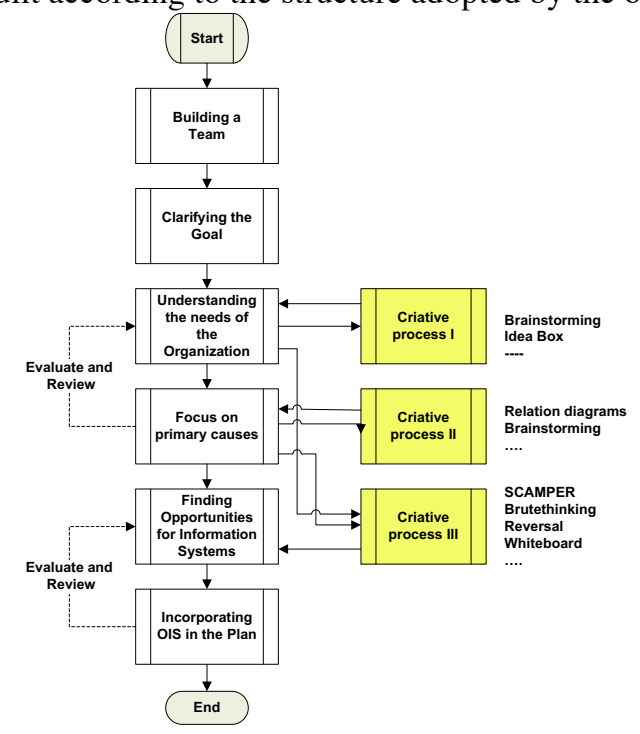

Figure 7 - Overview of ISP introduction and creativity generic method

In order to support the strategy application and the methods described above, we developed a series of tools and templates. In Figure 8, as an example, is one of the templates we developed.

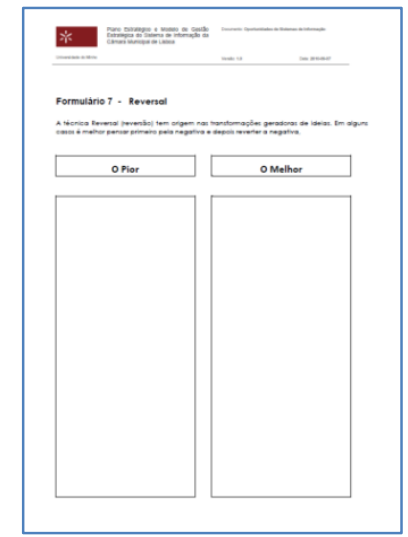

Figure 8 - Template for Reversal technique 
Some of the tools have for goal helping to the analysis of the problems, and data collection, others, to support the documentation production.

\section{$4 \quad$ Identification of Information Systems Opportunities in Câmara Municipal de Lisboa}

In the context of the Strategic Plan and Strategic Management Project for the Information System of Câmara Municipal de Lisboa (CML), several work sessions were made with the purpose of identifying innovative Information Systems Opportunities (ISO).

The first work session had as goal to identify innovative ISO which could help the Direcção de Património Cultural (DPC) solve one of the problems it faces in its mission. The session was 4 hours long.

The following method was used, as described below:

1- The team was formed by CML before initializing the session. A heterogeneous group was one care, with both experts and non-experts in the area of culture, so that would provide «out of the box» thinking. Apart from the people in the culture area (from DMC), the session was attended by services and informatics people.

2- The Direcção de Património Cultural (DPC) presented the following need of business:

«There is a need to improve the communication on cultural activities with the mayors and reach out to a new public. The media don't care there is dispersion of their means and excess of information».

The problem was broken down by filling a support paper and discussing and defined what the ideal solution would be (goal).

3- There was then a debate in order to identify and list the primary sources. The relations diagram is shown on Figure 9.

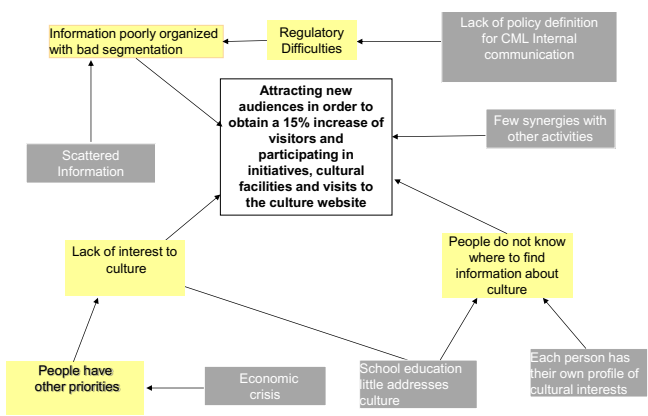

Figure 9 - Relation diagram

4- Based on what the ideal solution would be (goal), there was an identifying and a listing of key-points that would sustain an ideal solution. The primary causes list is shown on Figure 10. 
Figure 10 - Root cause list form

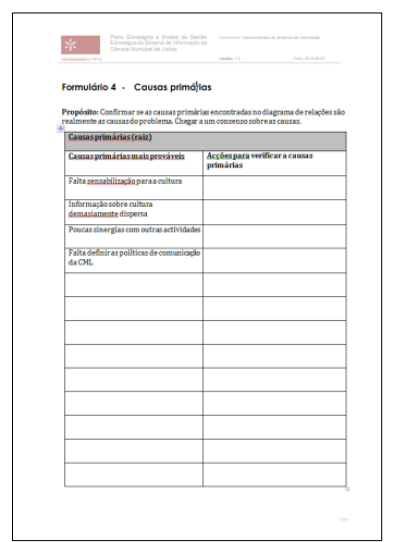

5- To approach the problem and according to the typology of the identified primary causes, two creative techniques were chosen and implemented: Brute Thinking and the Reversal.

Brute Thinking by Michael Michalko [10] is a creative and lateral thinking aid technique. It may be used to conceive alternate solutions, but also useful to identify problem causes.

This technique is based on a very simple process that is developed in four steps: to chose a random word; choose things/elements associated to the word randomly obtained; forcing a link between the word and the problem and between the associations and the problem; listing the ideas obtained and analyzing them.

The Reversal technique has its origin in the idea generating transformations included in the verification list of Osborn [11].

In some cases it is best to think first on the negative side then reverse the negatives.

6- From the application of both techniques came the seven following new ISO:

- CRM for Culture

- A publishing tool on City Cultural activities with a senior editor

- Culture and engineering synergy (culture and engineering portal)

- Culture Passport (every citizen can make a virtual collection of the events they attended, by the means of a stamp on their cultural passport- like in expo)

- Cultural offer Virtualization (virtual reality)

- Superculture Portal (all cultural activities information on a single website)

- Cultural program in terms of proximity (Project Unit or City zones)

DMC experts that attended declared that all the ISO found due to the creative techniques application are pertinent and promising.

\section{Conclusions}

Information Systems Planning is probably one of the most daring areas in Information Systems management. In a market environment characterized by the fast development 
of technologies and global competition intensification, in our view, the introduction of more creativity in the Information Systems Planning is of growing importance and has a probable impact in the success of Organizations.

The necessity for creative approaches in the design of new systems is both an opportunity and a challenge for Information Systems managers [5].

In this article we present a method for the introduction of creativity in the Information Systems Planning project, by describing it in a macroscopic level, along with a success case in its practical application in the context of information systems opportunities for CML. Applying the method resulted in seven new ISO.

So, in our opinion, it is possible to draw a consistent strategy for creativity and innovation processes in the Information Systems Planning, and make this work with simple and practical methods.

The next stages to be developed, following this work have mainly to do with the developing of theoretical models that support the design and developing of the strategy, perfecting the method, refining validation criteria and applicability analysis, and doing new tests and evaluations, as well as implementing eventual corrections.

\section{References}

1. Allaire, Y. and M. E. Firsitoru, "Coping with Strategic Uncertainty", Sloan Management Review, 30,3 Spring, 1989, 7-16.

2. Amaral, L. "PRAXIS: Um referencial para o Planeamento de Sistemas de Informação," in: Departamento de Sistemas de Informação, Universidade do Minho, Guimarães, 1994.

3. Amaral, L., and Varajão, J. Planeamento de Sistemas de Informação, FCA - Editora de Informática, Lda, Lisboa, $4^{\text {a }}$ ed, 2007, p. 247.

4. Bowman, B., G. Davis and J. Wetherbe, "Three Stage of MIS Planning" Information and Management, 6, 1, 1983.

5. Cooper, R.B. Information technology development creativity: A case study of attempted radical change. MIS Quarterly 24(2), 245-275, 2000.

6. Couger, J. D., "Ensuring Creative Approaches in Information System Design", Managerial and Decision Economics, 11, 1990, 281-295.

7. Earl, M. Management Strategies for Information Technologies Prentice Hall, London, 1989.

8. Horton, K.S., and Dewar, R.G. "Evaluating Creative Practice in Information Systems Strategy Formation: the application of Alexandrian patterns," 34th Hawaii International Conference on System Sciences, 2001.

9. Lederer, A. L, and V. Sethi, "Critical Dimensions of Strategic Information Systems Planning", Decision Sciences, 22, 1991, 104-119.

10. Michalko, Thinkertoys: A Handbook of Creative-Thinking Techniques (2nd Edition), Ten Speed Press, Toronto, 2006

11. Mycoted "Creativity, Innovation, Tools, Techniques, Books, Discussions, Puzzles, Brain Teasers, Training ..., 2011.

12. Ruohonen, Mikko \& Higgins, Lexis F. Application of Creativity Principles to IS Planning. In Hugh J. Watson (ed.) Proceedings of the Thirty-First Hawaii International Conference on System Sciences. Volume VI: Organizational Systems and Technology Track. Los Alamitos, California: IEEE Computer Society, 1998. 